\title{
Fixation instruction influences gaze cueing
}

\author{
Frouke Hermens \\ School of Psychology, University of Aberdeen, UK
}

\begin{abstract}
Studies have shown that perceiving another person's gaze shift facilitates responses in the direction of the perceived gaze shift. While it is often assumed that participants in these experiments remain fixated on the cue in the cueing interval, eye gaze is not always recorded to confirm this. The data presented here suggest that the effect of gaze cues on responses to peripheral targets depends on whether participants make eye movements prior to the onset of the target. Participants who were required to fixate, showed cueing effects at short cue-target intervals, but no cueing at later intervals. Participants who could look around, often chose to do so, and showed the same positive cueing effects at the shorter interval, but negative cueing effects (suggestive of inhibition of return) at the longer interval.
\end{abstract}

Keywords: Social attention, eye movements, inhibition of return, fixation control.

Several studies have demonstrated that perceiving another person's gaze shift or averted gaze can result in a strong shift of attention in the observer (e.g., Bayliss, Pellegrino, \& Tipper, 2004, 2005; Driver et al., 1999; Friesen \& Kingstone, 1998, 2003a, 2003b; Friesen, Ristic, \& Kingstone, 2004; Quadflieg, Mason, \& Macrae, 2004; Ristic, Friesen, \& Kingstone, 2002; Schuller \& Rossion, 2005; Senju, Tojo, Dairoku, \& Hasegawa, 2004). In these studies, an image or line-drawing of a face with an averted gaze is shown in the centre of the screen and, after an interval, a visual target (e.g., a dot or a letter) is presented in the direction of the averted gaze or in the opposite direction. Participants are asked to respond to the target as quickly as possible by responding to its location (e.g., by making an eye movement to that location, or pressing one of two response keys) or to the identity of the target (e.g., distinguish between two different letters). Gaze cues in these experiments are often unpredictive (meaning that the gaze cue is equally often directed towards the future target location as the opposite location, most studies) or counter-predictive (meaning that the gaze cue is more often directed away from the future target location than not, as in Driver et al., 1999; Friesen et al., 2004). Under both conditions, responses to the target are typically faster when the direction of the gaze cue is congruent with that of the target than when the direction of the cue is incongruent. For long intervals between the onset of the cue and the target,

The author wishes to thank Robin Walker for helpful discussions regarding the setup of the experiment. This study was supported by funding from the Research Foundation Flanders (FWO) in the form of a Postdoctoral Researcher grant to the author. The experiments were conducted while Frouke Hermens was at the University of Leuven, Belgium. Frouke Hermens is now in the School of Psychology, University of Lincoln, UK. This is an author's preprint. For the official version go to http://www.tandfonline.com/doi/abs/10.1080/13506285.2015.1042539 however, the congruency effect appears to reverse, and incongruent gaze cues lead to faster responses than congruent cues (after 2400ms in Frischen \& Tipper, 2004; Frischen, Smilek, Eastwood, \& Tipper, 2007; Marotta et al., 2013; Nestor, Klein, Pomplun, Niznikiewicz, \& McCarley, 2010 and after $1005 \mathrm{~ms}$ in Okamoto-Barth \& Kawai, 2006, but no such reversal was found by McKee, Christie, \& Klein, 2007). This reversal is known as inhibition of return (IOR, Klein, 2000) and is considered an important feature of stimuli that induce an exogenous shift of attention in the observer, contrasting with endogenous attention shifts typically following centrally presented symbolic cues (such as arrows, Müller \& Rabbitt, 1989). It may be questioned, however, whether IOR for gaze cues has the same underlying mechanism as for sudden onsets, where IOR is typically found at much shorter cue-target intervals (e.g., 300ms, Posner \& Cohen, 1984). Inhibition of return has also been reported for other types of centrally presented cues (arrows and words, Taylor \& Klein, 2000; Weger, Abrams, Law, \& Pratt, 2008), suggesting that IOR can also be found for cues normally considered to induce endogenous shifts of attention.

In most gaze cueing studies, participants were asked or instructed to maintain fixated on the cue until target onset (e.g., Bayliss et al., 2004, 2005; Friesen \& Kingstone, 1998, 2003a, 2003b; Friesen et al., 2004; Quadflieg et al., 2004; Schuller \& Rossion, 2005), although there are studies that do not make explicit mention of fixation instructions to the participant (e.g., Driver et al., 1999; Ristic et al., 2002; Senju et al., 2004). A possible reason for asking participants to maintain fixation is to ensure that the cue is being processed. Another reason may be to avoid influences of eye movements away from a possible target location, possibly leading to inhibition of return, particularly if followed by an additional eye movement bringing gaze back to the centre of the display (Taylor \& Klein, 2000). In one study (Friesen et al., 2004), the ability of participants to follow fixation instructions in a gaze cueing experiment was tested in a subset of participants, by measuring their eye movements and making participants 
aware that their eye movements were monitored. This subset of participants made few eye movements and their pattern of results in the response times was found to be indistinguishable from those participants whose eye movements were not monitored, suggesting that participants maintain fixated on the location of the cue when instructed to do so. Two other studies that monitored eye movements under fixation instructions reached similar conclusions. Mansfield, Farroni, and Johnson (2003) found that participants sometimes made eye movements in the direction of the cue, but found no differences in cueing between trials with or without such eye movements. Okamoto-Barth and Kawai (2006) found that participants remained fixated on the cue when instructed to do so.

While this small subset of studies measured participants' eye movements, the majority of gaze cueing experiments are conducted without eye movement monitoring. Fixation instructions appear to vary across studies and it is unclear whether each type of instruction leads to similar compliance with the fixation instruction. Examples of instructions include "stressing" to participants the importance of maintaining fixation (Friesen et al., 2004), "asking" participants to maintain fixation (Bayliss et al., 2004), and "instructing" participants to keep fixated (Friesen \& Kingstone, 2003a). It unclear whether in these studies participants were left on their own, and whether participants left on their own without mention of explicit checking of fixation maintain fixation during the relevant intervals in the experiment. While studies have ensured that participants maintain fixation by means of eye tracking, no studies appear to have investigated whether the results are in any way influenced by whether participants decide to move their eyes. With eye tracking, participants follow fixation instructions (Friesen et al., 2004; Mansfield et al., 2003; Okamoto-Barth \& Kawai, 2006), but this could be a consequence of participants knowing that their eye movements are monitored. No influences of incidental eye movements (Mansfield et al., 2003) and eye tracking (Friesen et al., 2004) were found under such conditions, possibly because fixation does not influence the cueing effect (Mansfield et al., 2003's results relied on a small number of observations and only considered small eye movements), or because participants automatically fixate when asked to do so. It is unclear what eye movement strategy participants adopt if not specifically instructed to maintain fixation. This no-fixation situation resembles natural vision more closely, in which there is no one to provide instructions on fixation. It is therefore important to establish whether the situation in the lab, where fixation instructions are the norm, resemble the situation it is trying to examine (i.e., how the human brain works outside the lab). Moreover, it is beneficial to establish possible effects of participants not following fixation instructions, particularly for research with research participants who may have difficulties maintaining fixation, such as children (e.g., Ristic et al., 2002), patient groups (e.g., Vuilleumier, 2002), or elderly (e.g., Slessor, Phillips, \& Bull, 2008; Slessor, Laird, Phillips, Bull, \& Filippou, 2010), because it would inform whether eye movements during the presentation of the cue influence the effects the cues has on participants' responses.

The present study examined the consequences of fixation instructions by tracking participants' eye movements in a gaze cueing experiment under varying fixation instructions. Because of the debate about the special status of social cues over symbolic cues (Friesen \& Kingstone, 1998; Ristic et al., 2002; Tipples, 2008) and findings suggesting that gaze cues lead to (unwanted) eye movements in the observer (Kuhn \& Kingstone, 2009; Kuhn, Tatler, Findlay, \& Cole, 2008; Kuhn, Tatler, \& Cole, 2009), the present study focuses on these types of cues, but the results should also apply to other types of cues (e.g., symbolic) and other paradigms (e.g., masked priming). In the experiment, participants were presented with a gaze cue in the centre of the display, showing an actor shifting his gaze leftwards or rightwards, superimposed by a small fixation target (Figure 1a). The cue was followed by a peripheral target either in the direction of the perceived gaze shift (congruent trials, $50 \%$ of the trials) or away from it (incongruent trials, the remaining $50 \%$ of the trials). Participants were randomly assigned to one of three groups. Two groups were asked to maintain fixation on the fixation target superimposed on the cue, and were informed that their eye movements would be recorded and monitored by the experimenter. In these groups, feedback was provided by the experimenter in the case of an eye movement to ensure that participants did not move their eyes during the cueing interval. Participants in the first group (eye movement response group) were asked to look at the fixation target in front of the cue until the appearance of the target inside one of two placeholders and then to look at the peripheral target as quickly as possible. The second group (fixation-controlled manual response group) were asked to maintain fixation at the fixation target until the appearance of the target and to press one of two keys as quickly as possible to indicate the location of the target. A third group (free viewing manual response group) did not receive fixation instructions. Instead, they were told that they were free to make eye movements if they felt this would help perform the task more quickly and more accurately. These participants were asked to respond to the location of the target by pressing one of two buttons as quickly and accurately as possible.

To increase the likelihood that participants would make eye movements during the cueing interval when allowed to do so, a dynamic cue was used, consisting of three successive frames from a movie clip showing an actor shifting his gaze. To examine whether cueing effects for this cue followed the direction of the gaze shift and not solely the perceived direction of motion, the gaze shift was accompanied by an oppositely directed head position shift in half of the trials (see also, Bayliss et al., 2005), causing the pupil location to be stationary with respect to the background. To examine the temporal dynamics of any differences between congruent and incongruent trials, three stimulus onset asynchronies (the time between the onset of the gaze cue and the onset of the target) were used, consisting of a short (100ms), medium (500ms), and long $(900 \mathrm{~ms})$ interval. To anticipate the results, the majority of participants chose to make eye movements when allowed to do so, shifting their gaze between the two 
possible target locations and the center of the display. Differences in the time-course of the cueing effects (the difference between congruent and incongruent trials) were found between the fixation and no-fixation groups, with evidence of inhibition of return (longer response times for congruent conditions than for incongruent conditions) at longer intervals in the no-fixation group. By analyzing the timing of the eye movements with respect to the cueing effects, the possible source of these effects was investigated.

\section{Methods}

\section{Participants}

A total of 57 students from the University of Leuven (52 female, aged between 17 and 23 years) took part in return for course credit. Twenty participants performed the eye movement task, seventeen performed the fixation-restricted button press task, and twenty performed the button press task without fixation restriction.

\section{Apparatus}

Stimulus presentation was controlled by a Pentium PC, using a NVIDIA GeForce 7600 GT graphics card, while a second PC (LanBox Lite) controlled the measurement of the eye movements. Stimuli were presented on a 22 inch CRT monitor (Iiyama HM204DT A) at a $75 \mathrm{~Hz}$ refresh rate and in a $1024 \times 768$ pixels spatial resolution. Eye movements were recorded using an Eyelink 1000 system with a desk mount setup. A chin rest, placed at $60 \mathrm{~cm}$ from the screen, was used to control to distance to the screen and to avoid head movements in the participants that could interfere with eye tracking. Manual responses were recorded using a Microsoft Sidewinder gamepad connected to the USB port of the LanBox PC.

\section{Stimuli}

The stimulus sequence is illustrated in Figure 1a. A fixation cross (a plus sign measuring 0.39 by 0.39 degrees of visual angle) was presented in the middle of the screen, which remained in place until the end of the trial. $800 \mathrm{~ms}$ to $1200 \mathrm{~ms}$ after onset of the fixation cross, a picture of a face and two place-holders appeared. The image of the face was placed behind a frame in the same color as the background (zero contrast, black), so that the visible part of the image measured 10.4 by 5.22 degrees. This frame was used so that the image could be moved in the opposite direction of the gaze shift without a visible shift of the edges of the image (actually, many participants did not report seeing the shift of the head position when asked about this at the end of the experiment). The place-holders measured 0.74 degrees in diameter and were placed at the horizontal midline at a distance of 8.8 degrees from the centre of the screen. After an SOA of $500 \mathrm{~ms}$, the face in the image shifted his gaze (complemented by an opposite shift of the image in half of the trials) across three frames (with a total duration of approximately $40 \mathrm{~ms}$, each of the three frames lasted approximately $13.3 \mathrm{~ms}$ ). A target consisting of a plus sign measuring 0.7 by 0.7 degrees appeared inside one of the place-holders after an SOA varying between $100 \mathrm{~ms}, 500 \mathrm{~ms}$ and $900 \mathrm{~ms}$.

\section{Design}

Participants performed two blocks of 96 trials each, resulting in a total of 192 trials. For each group of participants four factors were varied: The location of the target (left or right), the direction of the perceived gaze shift (left or right), whether the pupils were stationary or moved with the gaze shift (Figure 1b), and the stimulus onset asynchrony $(100 \mathrm{~ms}$, $500 \mathrm{~ms}$, or $900 \mathrm{~ms}$ ), resulting in 24 combinations, which were each presented 8 times to each participant. In the data analysis, data were pooled across left and right targets and left or right perceived gaze shifts, by separating congruent targetgaze and incongruent target-gaze combinations. Each of the 12 resulting combinations (congruency $\mathrm{x}$ pupil stationarity $\mathrm{x}$ SOA) was therefore tested 16 times per participant. Because each combination of conditions was presented equally often, the gaze cue was unpredictive of the future location of the target. The order of the trials was randomized for each participant.

\section{Procedure}

Before taking part, participants signed an informed consent and received verbal instructions about the task. They then took place in the chin rest, holding the gamepad in their hands if taking part in one of the button press conditions of the experiment. The experiment started with the calibration of the eye tracker for all participants, meaning that participants were aware that their eye movements were tracked. The standard nine-point calibration procedure (targets presented on a three by three grid) of the Eyelink 1000 system was used. Calibration was accepted if the recorded fixations were on a similar three by three grid as the fixation targets, or repeated otherwise. During the experiment, drift correction was applied after every 12 th trial.

Participants then received a few practice trials to get used to the task. Depending on the group they were assigned to, they were required to maintain fixation until the appearance of the target stimulus or were free to move their eyes. Also depending on the group, participants responded by means of an eye movement, or with a button press.

Participants each performed 192 trials (Figure 1a). Trials started with a fixation cross for a duration between $800 \mathrm{~ms}$ and $1200 \mathrm{~ms}$, followed by a preview of the face looking straight ahead for $500 \mathrm{~ms}$, a gaze shift for $40 \mathrm{~ms}$, and after an SOA (of $100 \mathrm{~ms}, 500 \mathrm{~ms}$ or $900 \mathrm{~ms}$ ) followed by the presentation of the target until the button press, a 3000ms timeout or for $1000 \mathrm{~ms}$ in the eye movement group. Each trial ended with a $200 \mathrm{~ms}$ blank screen. After completing the experiments, participants were debriefed about the purpose of the experiment. The experiment took approximately $25 \mathrm{~min}$ utes for each participant to complete. 
a) Stimulus sequence

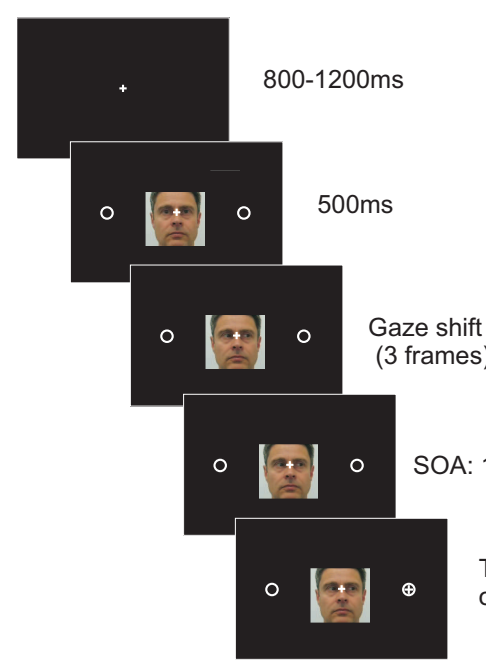

b) Gaze shift conditions

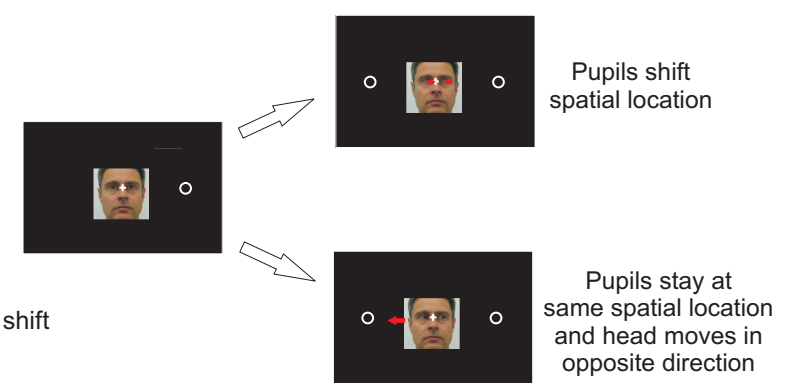

Figure 1. a) Illustration of the stimulus sequence. A fixation cross was followed by the image of a person looking straight ahead. After an stimulus onset asynchrony (SOA) of 500ms, the person shifted his gaze to the left or right. In the "head shift" condition, this gaze shift was accompanied by a shift of the entire head in the opposite direction, such that the pupils remained stationary with respect to the background. After an SOA of $100 \mathrm{~ms}, 500 \mathrm{~ms}$ or $900 \mathrm{~ms}$, a visual target appeared inside one of the two place-holders. Different groups of participants received different instructions. The first group was required to maintain fixation until target appearance, and make an eye movement to the target. The second group received the same fixation requirement, but responded by pressing one of two buttons to indicate the location of the target. The third group was free to look around, and was asked to respond to the target location by pressing one of two response keys. b) Illustration of the two stimulus conditions, in which a gaze shift was presented on its own, or with a simultaneous shift of the head in the opposite direction.

\section{Data analysis}

For the analysis of the eye movements in all three participant groups, the raw eye movement signal was parsed into fixations and saccades by using the eye tracker's parser, applying the default combined $30 \mathrm{deg} / \mathrm{sec}$ velocity and 8,000 $\mathrm{deg} / \mathrm{sec}^{2}$ acceleration detection criterion. Response times in the eye movement group were defined as the time between the onset of the target and the onset of the saccade. Correct responses were defined as saccades of at least 5.8 degrees in the target's horizontal direction and of less than 4 degrees in the vertical direction (allowing for some error in the vertical aiming of the saccade). Responses faster than $80 \mathrm{~ms}$ (anticipations) and slower than the participant's overall mean response time plus 2.5 times their standard deviation were excluded as outliers. Trials in which a blink occurred during the saccadic eye movement were also removed. One participant, for whom more than $30 \%$ of the trials needed to be removed on the basis of these criteria, was removed from the analysis. For the remaining 19 participants, an average of $8.9 \%$ of the trials were excluded.

The same response time outlier procedure was used for button press responses (meaning that responses faster than $80 \mathrm{~ms}$ and slower than the mean plus 2.5 times the standard deviation for that participant were excluded). Incorrect button presses were also removed. Error rates were lower in the button press tasks (on average $2.6 \%$ with fixation control and $3.6 \%$ without), and therefore no participants were removed from these conditions. Because fixation was monitored during the experiment, and participants were immediately corrected when not maintaining fixation during the cueing interval, failures to maintain fixation (if instructed so) were sporadic, and no filtering was conducted for this aspect of the data.

\section{Results}

The majority of participants in the group without fixation instruction chose to make eye movements. Of the twenty participants in this group, only three of the participants chose to maintain fixation on the fixation point from the start of the experiment. However, six further participants who started making eye movements at the beginning of the experiment later decided to stop making eye movements and maintain fixation on the fixation point instead. For the present analysis, the data of all participants within this group were pooled (to test the effect the instruction, rather than behaviour) and compared to those of the two groups with fixation instruction, although for some important effects, mention will be made of the results without the participants who chose to maintain fixation in part of the experiment.

The main results are shown in Figure 2, where the mean 
response times for each of the groups are shown as a function of the stimulus onset interval between the cue and the target, split between congruent and incongruent trials and eyesgaze shifts and opposite head-shifts (with stationary pupils). The data show a positive cueing effect (faster response times in the congruent than in the incongruent conditions) for the shorter $100 \mathrm{~ms}$ stimulus onset asynchrony in all three instruction groups, except for the eyes-gaze shift stimulus condition without fixation instruction, where the p-value falls short of the Bonferroni corrected criterion of 0.0083 (corrected for six comparisons per participant group; $100 \mathrm{~ms}, 500 \mathrm{~ms}$ and $900 \mathrm{~ms}$ SOAs for eye shifts and head shifts). At an SOA of $500 \mathrm{~ms}$, none of the comparisons between congruent and incongruent conditions survives Bonferroni correction. After $900 \mathrm{~ms}$, the cueing effect vanishes or reverses. In particular, without fixation instruction, trials in which the gaze cue was provided by a gaze shift showed faster response times on incongruent trials than on congruent trials (a reverse cueing effect, suggestive of inhibition of return). The same trend can be observed for the head-shift in this group, where the difference just falls short of significance under (the rather stringent) Bonferroni correction.

In a subsequent analysis, the eye movements of the group of participants without fixation instruction are analyzed to gain a better understanding of why the effects of the cues on response times are different in this group. To illustrate the analysis, Figure 3a provides an example of a scanpath of one of the participants, typical of the eye movements made by the participants in the no-fixation instruction group. On the horizontal axis of this plot, the horizontal position of the eye on the screen is shown, with the dashed lines indicating the positions of the place-holders and the fixation point. The vertical axis represents the time after fixation onset. The horizontal lines in the plot indicate the moments on which the fixation point, the face, the gaze shift and the target were presented. The scanpath shows that, on this particular trial, the participant is fixating slightly right of fixation before fixation onset. After fixation onset, a saccade to the fixation point is made. During preview (face without a gaze shift), an eye movement to the right place-holder is made, but as soon as the gaze cue is presented, the participant makes an eye movement in the direction of the cue. At target onset the participant is still looking at the left place-holder, but an eye movement to the right is made before the response. To obtain an impression of where participants in the group without fixation instruction were looking, Figure $3 \mathrm{~b}$ plots all fixation locations between fixation onset and the response. This plot shows that the majority of fixations are on the two placeholders (left and right) and the gaze cue (in the centre). The vertical deviations in the recordings of the eye movements may reflect actual vertical gaze shifts, or measurement error (for the eye tracking system used, accuracy in the horizontal direction is typically better than the vertical direction). Figure $3 \mathrm{c}$ shows that most trials in which participants chose to look around, involved fixations on all three regions of interest (left place-holder, cue in the centre, and right place-holder).

In order to examine how the eye movements in the group without fixation instruction influence response times, the fix- ation locations at target onset were classified into eye movements on the left place-holder, the right place-holder, and the centre of the screen. Regions of interest were defined by dividing the screen into three areas with gaze positions more than 100 pixels left of the centre assigned to the left place-holder, fixations more than 100 pixels to the right of the centre assigned to the right place-holder and the remaining gaze positions assigned to the centre of the display (blue lines in Figure $3 \mathrm{~b}$ showing the boundaries of the areas). Prior to the analysis data from one participant had to be excluded, whose eye movement data were not correctly stored (but for whom eye movements were actively monitored during the experiment). Two comparisons were made: One in which fixation at target onset is related to the direction of the cue (to examine whether participants followed the cue and remained fixated on the relevant place-holder), and one in which fixation at target onset is related to the position where the target appeared (to examine whether responses are faster when already fixating the location where the target appeared).

Figure $3 \mathrm{~d}$ shows where participants fixate at target onset relative to the direction of the cue. This data plots suggests that participants equally often look at the place-holder gazed at by the cue as the opposite place-holder (all p-values larger than 0.05; similar findings are obtained when excluding participants choosing not to make eye movements at some point in the experiment). Figure 3e examines how often participants fixated the place-holder in which the target appeared. Because the conditions were fully randomized and the cues were $50 \%$ valid, participants had no way of predicting where the target was going to appear (in the cue direction or not), and therefore it is expected that participants equally often look at the place-holder with the target as the place-holder without the target. This prediction is confirmed: There was no difference in how often the two place-holders were fixated relative to the target location (all p-values $>0.075$ ).

Figure $3 \mathrm{f}$ plots response times dependent on where participants fixated at target onset, with respect to where the target appeared. Response times were faster when the target location was fixated, compared to the other two positions $\left(\mathrm{F}(1,18)=9.65, \mathrm{p}=0.006, \eta_{p}=0.35\right.$; for the three participants in the analysis who chose to remain fixated, the empty cells for the two place-holder positions were replaced by the mean of the other participants, similar results are obtained if these three participants are excluded from the analysis). Responses for trials on which participants fixated the centrally presented cue at target onset showed a similar pattern of results as for the two groups who were instructed to fixate the cue, but paired comparisons no longer revealed significant differences between congruent and incongruent cue conditions (all p-values $>0.43$ ). When participants fixated the opposite place-holder from where the target appeared at target onset, incongruent trials had faster response times than congruent trials, but only at the longer SOA (900ms, $\mathrm{p}=0.0017)$.

\section{Discussion}

Studies examining gaze cueing often provide fixation instructions to participants without eye gaze being tracked. 


\section{Eye movement task $(\mathrm{N}=19)$}
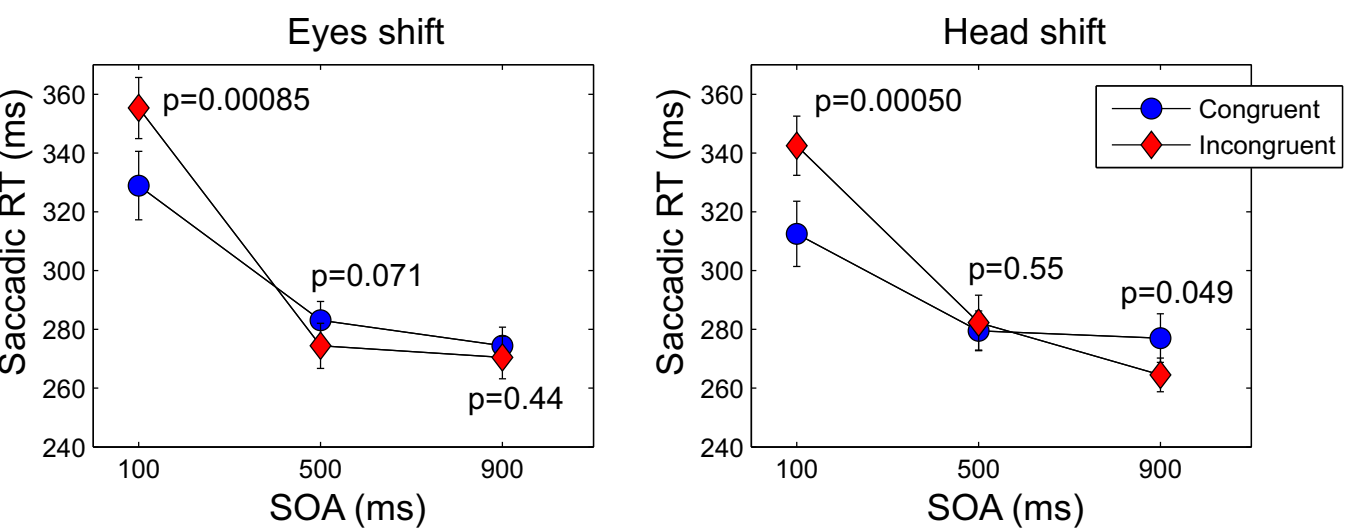

\section{Button press with fixation $(\mathrm{N}=17)$}
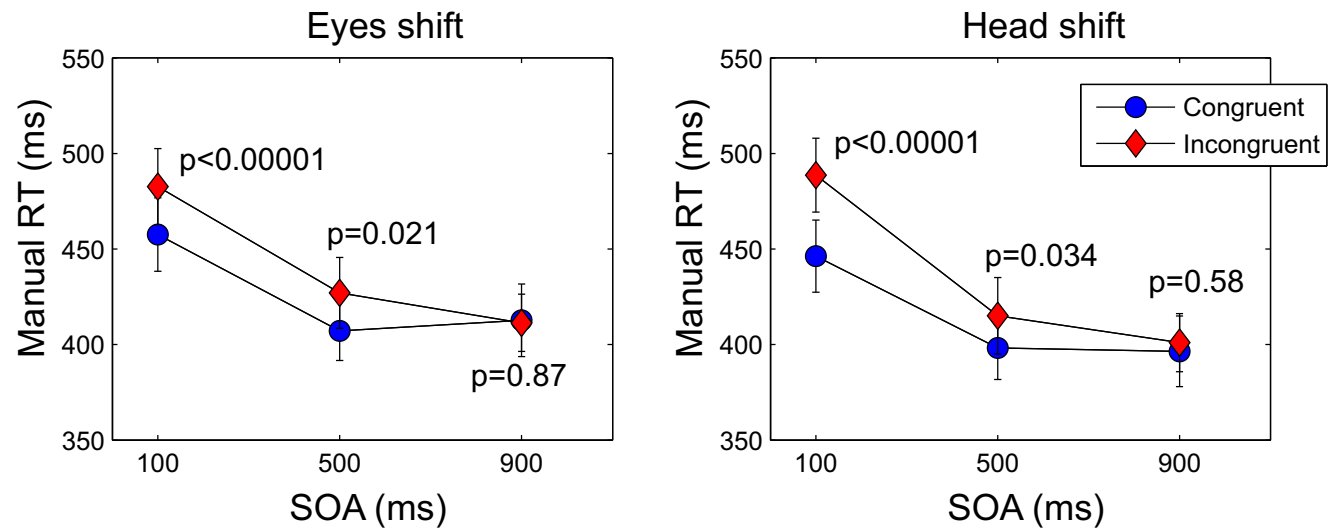

\section{Button press without fixation $(\mathrm{N}=20)$}
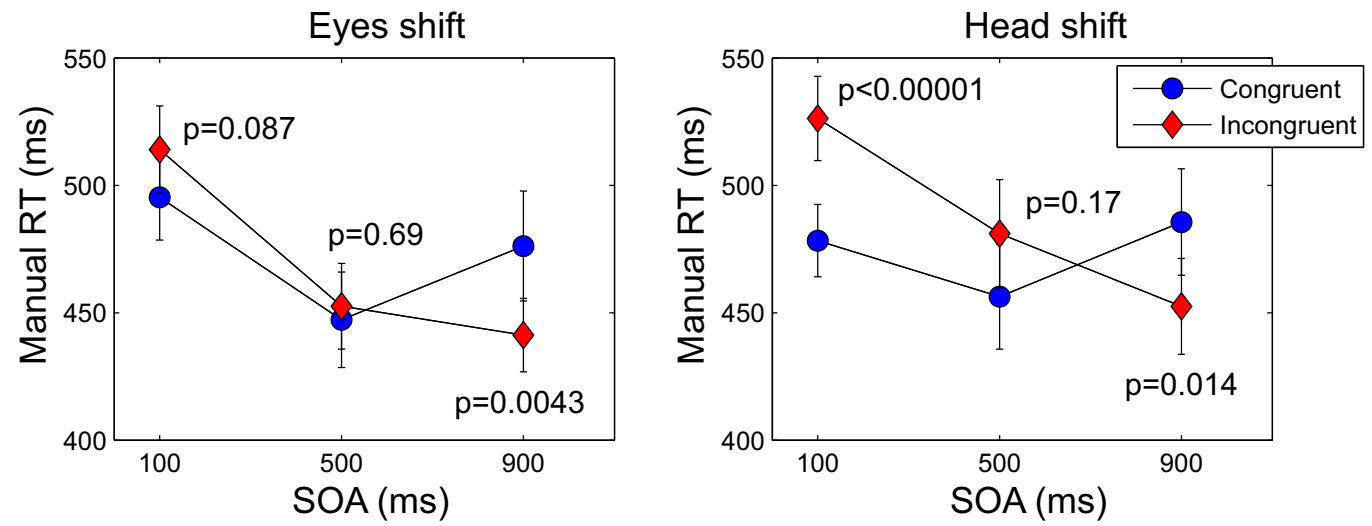

Figure 2. Overview of the mean response times (after filtering for error responses and outlier data) across the three participant groups. Response times (in ms) indicate the time until button press or saccade initiation, and are plotted as a function of the time between the onset of the perceived gaze shift and the target (SOA $=100 \mathrm{~ms}, 500 \mathrm{~ms}$ or $900 \mathrm{~ms}$ ). The left subplot shows the data for the gaze shift in isolation, and the subplot on the right for the gaze shift accompanied with the opposite head shift (leaving the pupils stationary on the screen). (Blue) circles show response times for congruent gaze shifts and targets, whereas the (red) diamonds indicate the response times for the incongruent conditions. Error bars show the standard error of the mean across participants and p-values the outcome of paired samples t-tests between congruent and incongruent conditions. Under a Bonferroni correction for six comparisons per participant group, only p-values smaller than 0.0083 can be considered statistically significant. 


\section{a) Scanpath example}

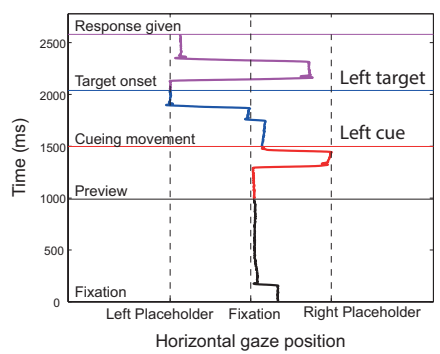

b) Distribution of fixations

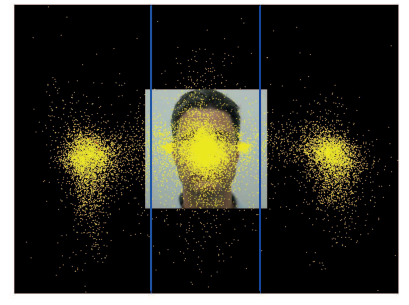

c) Inspected regions

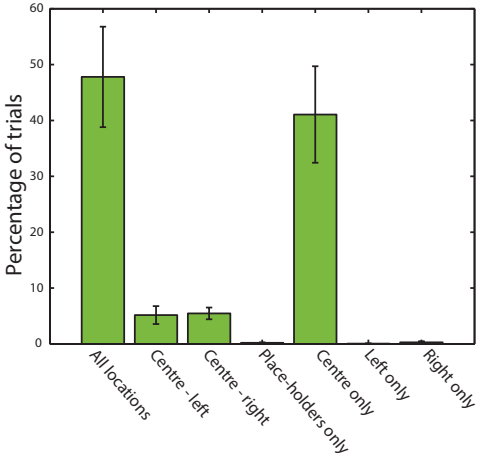

d) Cue-directed fixations
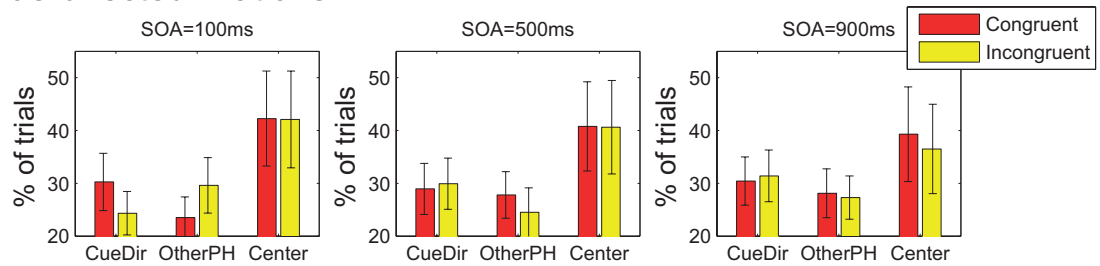

e) Target-direct fixations
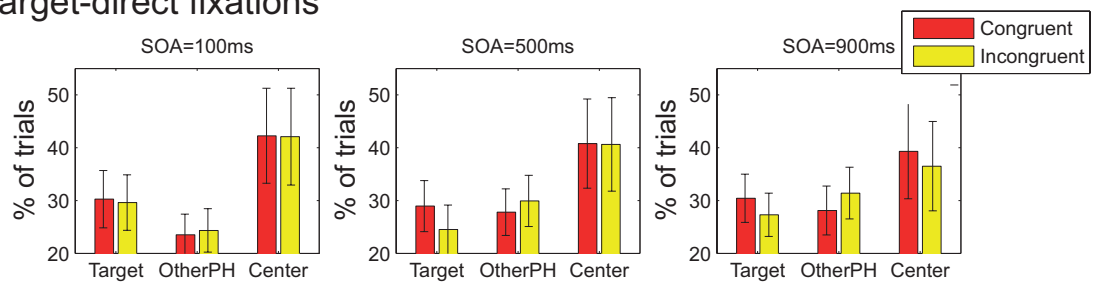

\section{f) Response times}
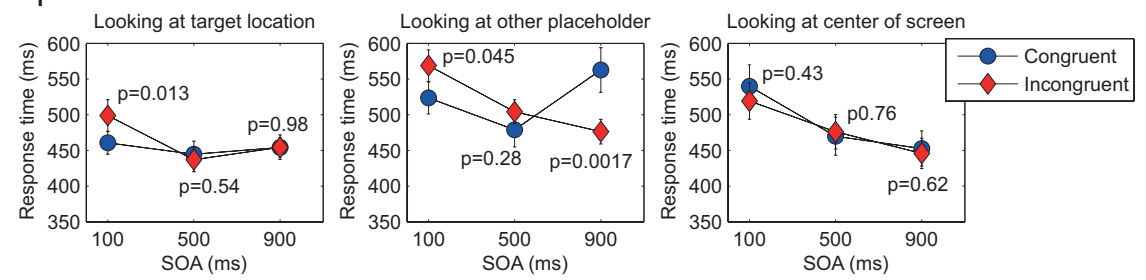

Figure 3. a) Example of a scanpath, showing the participant's horizontal gaze position on the screen on the horizontal axis and time on the vertical axis. Horizontal lines indicate events, such as cue onset and target onset, whereas dashed vertical lines indicate the position of the place-holders and the fixation point. The example is for a leftward cue with a left target. b) Fixation locations across conditions and participants, showing that participants mostly fixated the centre of the screen, the left place-holder or the right place-holder. d) Percentage of trials with fixations on different combinations of regions, showing that participants predominantly chose to either fixate all three regions, or to fixate the centre region only. d) Percentage of trials in which the participant was fixating in the direction of the cue at the end of the cueing interval (i.e., at target onset). e) Percentage of trials in which the participant was fixating where the target appeared at target onset. f) Response times across trials in which participants looked at the target location (left plot), the other place-holder's location (middle plot), or at the fixation target (right subplot) at the end of the cueing interval (target onset). P-values are shown for paired samples t-tests comparing congruent and incongruent conditions. 
Studies in which eye movements were measured (Friesen et al., 2004; Mansfield et al., 2003; Okamoto-Barth \& Kawai, 2006) suggest that participants comply with a fixation instruction when their eye movements are tracked. Friesen et al. (2004) also showed that effects of gaze cues were independent of whether participants' eye movements were tracked. This could mean that participants comply with a fixation instruction even when knowing their gaze is not monitored, but it could also mean that gaze cueing effects do not depend on whether or not participants make eye movements. The present study examined whether participants decide to make eye movements when no fixation instruction is given, and how these (potential) eye movements influence the effects of gaze cues. The results show that (1) participants instructed to fixate, follow this instruction, and (2) their response times were faster for congruent cue-target combinations compared to incongruent cueing, but only at a short interval between cue onset and target onset (of 100ms). (3) Participants without fixation instruction often chose to make eye movements, and gazed between the centre of the screen, the left place-holder and the right place-holder (where the target could appear). (4) Participants not instructed to fixate showed the same congruency effect at the shorter (100ms) cue-target interval, but show a reversed cueing effect (suggestive of inhibition of return) at the longer SOA (900ms). (5) By analyzing where participants fixated at the moment the target appeared, it was shown that reverse cueing at this long 900ms SOA only occurred when participants were fixating the place-holder opposite to where the target appeared.

The present results confirm earlier findings that participants fixate when instructed to - when their eye movements are tracked. This does not automatically mean that participants whose eye movements are not tracked, and are possible left on their own during the experiment, also show the same behaviour (this would require the covert tracking of eye gaze, which, with the current state of technology, is more complicated). However, a case can be made that participants follow fixation instructions. The present results show that when participants were allowed to make eye movements, the majority chose to do so. Moreover, the effects of the social cues depended on the participants' eye movements, and in particular, on where participants looked at the moment the response target appeared. Combined with the results from Friesen et al. (2004), who found no differences in effects of cues between participants whose eye movements were tracked and those participants whose eye movements were not tracked (both groups were required to fixate), this suggests that participants follow fixation instructions. The present results may seem at odds with Mansfield et al. (2003)'s, who found no effect of accidental eye movements in the presence of gaze cues. However, the eye movements in their study appeared to be small in amplitude, and the conclusions were drawn on a relatively small sample of observations. A possible reason for the small eye movements in Mansfield et al. (2003) may be that the study did not make use of place-holders, which seem to have driven eye movements in the present study.

When participants were allowed to make eye movements, incongruent cues at the longer SOA $(900 \mathrm{~ms})$ led to faster re- sponses than congruent cues, a pattern of results congruent with inhibition of return (Klein, 2000). Analysis of the eye movements in this condition showed that the advantage for incongruent cues only occurred across trials in which participants fixated the opposite place-holder at the moment the target appeared. The faster response times to incongruent target-cue combinations in these trials were not an immediate consequence of fixating the place-holder where the target appeared, because faster incongruent responses were only found for a stimulus onset asynchrony of $900 \mathrm{~ms}$, and not for the shorter $100 \mathrm{~ms}$ and $500 \mathrm{~ms}$ SOAs. At these shorter SOAs participants were also fixating the place-holder with the target at target onset on incongruent trials. It has been suggested that inhibition of return serves the purpose of preventing revisiting an already inspected location during visual search (Klein, 1988; Klein \& MacInnes, 1999). Such an explanation would fit the current data, because in almost all trials in which participants fixated the opposite place-holder at target onset, they had also fixated the other place-holder (Figure $3 \mathrm{c}$ ), meaning that sometime during the trial, an eye movement had been made away from the target location. There is insufficient data to examine whether the reverse cueing effect was a direct consequence of fixating the target place-holder immediately before fixating the opposite place-holder, or to determine how much time can elapse between fixating the target place-holder and the opposite place-holder before the reverse cueing effect no longer occurs. This would be an interesting area of interest for future studies, which could focus specifically on data without a fixation instruction.

The inhibition of return observed in the present study occurs at a relatively short stimulus onset asynchrony (900ms), compared to previous studies that used social cues, where inhibition of return was only found after $2400 \mathrm{~ms}$ (Frischen \& Tipper, 2004; Frischen et al., 2007; Marotta et al., 2013; Nestor et al., 2010). Only one other study found IOR for social cues at a shorter SOA of $1005 \mathrm{~ms}$ (Okamoto-Barth \& Kawai, 2006), but the size of the effect was relatively small (for a comparison, see McKee et al., 2007). The present study only shows evidence of IOR when participants are allowed to make eye movements, and the eye movement analysis shows that IOR is found particularly when participants fixated away from where the target appeared, suggesting that the reason why the present study found evidence of IOR at a relatively short SOA was due to the eye movements. Interestingly, participants who chose to make eye movements, did so throughout the entire trial sequence and in almost all trials all three relevant areas on the screen (left place-holder, right place-holder and screen centre) were visited. At target onset, the place-holder fixated was unrelated to the direction of the cue. It is unclear why participants chose the eye movement strategy that they did. The use of place-holders may be important and it would be interesting to determine whether similar eye movement patterns would be found if no placeholders would be used.

The present study focused on gaze cues, but fixation instruction is likely to be important in other paradigms as well. For example, in masked priming (where a prime is masked immediately by a masking stimulus, but still exerts on in- 
fluence on a target stimulus) it is assumed that participants fixate on the area where the prime is presented. The subsequent presentation of the target at the same location makes it likely that participants do, but fixation is not typically verified by using an eye tracker. The same holds for cueing paradigms with other types of cues, for example using arrow signs. Inhibition of return has been found with these cues in conditions in which participants can be expected to prepare an eye movement in the direction of the cue (Taylor \& Klein, 2000), and under conditions where cued locations and predicted target locations are dissociated (Weger et al., 2008). Both studies (Taylor \& Klein, 2000; Weger et al., 2008) used eye tracking to ensure that participants fixated the cue during the cueing interval, and therefore cueing in these conditions cannot be attributed to eye movements made before target onset. These past demonstrations of IOR suggest that IOR can be found at shorter intervals and for symbolic cues, even under fixation instructions. It is unclear why the present data did not show IOR in the two fixation instruction groups. A possible reason could be that the past studies (Taylor \& Klein, 2000; Weger et al., 2008) have used stimuli at the centre of fixation after presentation of the cue, which may increase the chances of finding IOR. The demonstration of IOR for arrow cues and word stimuli in these studies suggests that IOR can be found for symbolic stimuli, casting doubt on the assumption that IOR is restricted to stimuli eliciting exogenous shifts of attention (arrows, and in particular words, are not normally assumed to induce such automatic shifts of attention, and instead lead to voluntary attention shifts). It is therefore unclear to which extent the present data contribute to the discussion on the type of attention (exogenous or endogenous) elicited by social cues (Friesen \& Kingstone, 1998; Langton, Watt, \& Bruce, 2000; Tipples, 2008).

The present work used two ways of presenting the dynamic gaze cue. These involved a normal shift of gaze in which the pupils in the actor's eyes physically moved and one in which the head was shifted in the opposite direction of the gaze shift, such that the position of the pupils remained stationary with respect to the background. The pattern of results in the two conditions was almost identical, suggesting that the cueing effects follow the direction of the gaze shift, rather than the direction of the motion field (see also Bayliss et al., 2005). Cues to the shift of the head were removed by moving the image behind a frame in the same colour as the background. Interestingly, many participants failed to report the two different conditions when asked about it at the end of the experiment (i.e., they did not see the head shift). In an earlier study, we did not use such a control condition (Hermens \& Walker, 2012) and therefore the present results are important in that they show that our past results were due to the perceived gaze shift and not due to the direction of perceived motion in the display.

Participants in the groups with fixation instruction responded either by making an eye movement to the target or by pressing a key to indicate the location of the target. These two response modes were introduced to examine whether any differences would be obtained between eye movement and button press responses. As expected, eye movement responses were faster than button press responses (see also Friesen \& Kingstone, 2003b). The pattern of results, however, was very similar across the two response modes. A weak trend towards inhibition of return was found for eye movements at the longer SOAs, but this effect did not reach statistical significance. This could be a power issue, and with additional participants the difference between congruent and incongruent cues may become significant. At the shorter 100ms SOA, large differences between congruent and incongruent cues were found, both for saccadic and manual responses. Such early effects of the cue have not been consistently observed. For example, using a static gaze cue, Driver et al. (1999) found no cueing at 100ms (but significant cueing at $300 \mathrm{~ms}$ or $900 \mathrm{~ms}$ ). Similarly, using a dynamic gaze cue Bayliss et al. (2005) only found cueing at longer SOAs, and only for female participants. In contrast, Langton and Bruce (1999) and Okamoto-Barth and Kawai (2006) found significant cueing at SOAs of $100 \mathrm{~ms}$ and $105 \mathrm{~ms}$, respectively. The late cueing effect in past studies has been hypothesized to be due to small eye movements made in the direction of the cue, which require time to initiate, but no evidence for such influence of eye movements on cueing was found (Mansfield et al., 2003). The present study did not find significant late cueing effects (at $500 \mathrm{~ms}$ or $900 \mathrm{~ms}$ ), but this could be a power issue, and with more participants, the smaller difference between congruent and incongruent trials (particularly at 500ms) may be picked up. Future studies should assess what factors determine whether cueing occurs at short or longer SOAs.

The importance of examining participants' eye movements during cueing experiments was also demonstrated by Burigo and Knoeferle (2014). In their study, differences in symbolic cueing were found between tight fixation control and more relaxed fixation control (allowing the wandering of gaze across a larger spatial window). Interestingly, in their study cueing was found only for relaxed fixation control, while in the present study cueing was found across both fixation conditions, particularly at the short SOA. The difference may originate in the difference in the stimuli used, with dynamic gaze cues leading to stronger cueing than static arrow cues, although this needs to be verified in future work.

\section{References}

Bayliss, A. P., Pellegrino, G. di, \& Tipper, S. P. (2004). Orienting of attention via observed eye gaze is head-centred. Cognition, 94(1), B1-B10.

Bayliss, A. P., Pellegrino, G. d., \& Tipper, S. P. (2005). Sex differences in eye gaze and symbolic cueing of attention. The Quarterly Journal of Experimental Psychology, 58(4), 631-650.

Burigo, M., \& Knoeferle, P. (2014). Keeping the eyes on a fixation point modulates how a symbolic cue orient s covert attention. In Proceedings of the 36th Annual Meeting of the Cognitive Science Society (pp. 278-283). Austin, TX.

Driver, J., Davis, G., Ricciardelli, P., Kidd, P., Maxwell, E., \& Baron-Cohen, S. (1999). Gaze perception triggers reflexive visuospatial orienting. Visual Cognition, 6(5), 509-540.

Friesen, C. K., \& Kingstone, A. (1998). The eyes have it! reflex- 
ive orienting is triggered by nonpredictive gaze. Psychonomic Bulletin \& Review, 5(3), 490-495.

Friesen, C. K., \& Kingstone, A. (2003a). Abrupt onsets and gaze direction cues trigger independent reflexive attentional effects. Cognition, 87(1), B1-B10.

Friesen, C. K., \& Kingstone, A. (2003b). Covert and overt orienting to gaze direction cues and the effects of fixation offset. Neuroreport, 14(3), 489-493.

Friesen, C. K., Ristic, J., \& Kingstone, A. (2004). Attentional effects of counterpredictive gaze and arrow cues. Journal of Experimental Psychology: Human Perception and Performance, 30(2), 319.

Frischen, A., Smilek, D., Eastwood, J. D., \& Tipper, S. P. (2007). Inhibition of return in response to gaze cues: The roles of time course and fixation cue. Visual Cognition, 15(8), 881-895.

Frischen, A., \& Tipper, S. P. (2004). Orienting attention via observed gaze shift evokes longer term inhibitory effects: implications for social interactions, attention, and memory. Journal of Experimental Psychology: General, 133(4), 516.

Hermens, F., \& Walker, R. (2012). Do you look where i look? attention shifts and response preparation following dynamic social cues. Journal of Eye Movement Research, 5(5).

Klein, R. (1988). Inhibitory tagging system facilitates visual search. Nature, 334(6181), 430-431.

Klein, R. M. (2000). Inhibition of return. Trends in Cognitive Sciences, 4(4), 138-147.

Klein, R. M., \& MacInnes, W. J. (1999). Inhibition of return is a foraging facilitator in visual search. Psychological Science, 10(4), 346-352.

Kuhn, G., \& Kingstone, A. (2009). Look away! eyes and arrows engage oculomotor responses automatically. Attention, Perception, \& Psychophysics, 71(2), 314-327.

Kuhn, G., Tatler, B. W., \& Cole, G. G. (2009). You look where i look! effect of gaze cues on overt and covert attention in misdirection. Visual Cognition, 17(6-7), 925-944.

Kuhn, G., Tatler, B. W., Findlay, J. M., \& Cole, G. G. (2008). Misdirection in magic: Implications for the relationship between eye gaze and attention. Visual Cognition, 16(2-3), 391-405.

Langton, S. R., \& Bruce, V. (1999). Reflexive visual orienting in response to the social attention of others. Visual Cognition, 6(5), $541-567$.

Langton, S. R., Watt, R. J., \& Bruce, V. (2000). Do the eyes have it? cues to the direction of social attention. Trends in Cognitive Sciences, 4(2), 50-59.

Mansfield, E., Farroni, T., \& Johnson, M. (2003). Does gaze perception facilitate overt orienting? Visual Cognition, 10(1), 7-14.

Marotta, A., Pasini, A., Ruggiero, S., Maccari, L., Rosa, C., Lupiáñez, J., \& Casagrande, M. (2013). Inhibition of return in response to eye gaze and peripheral cues in young people with aspergers syndrome. Journal of Autism and Developmental Disorders, 43(4), 917-923.

McKee, D., Christie, J., \& Klein, R. (2007). On the uniqueness of attentional capture by uninformative gaze cues: Facilitation interacts with the simon effect and is rarely followed by ior. Canadian Journal of Experimental Psychology/Revue canadienne de psychologie expérimentale, 61(4), 293.

Müller, H. J., \& Rabbitt, P. M. (1989). Reflexive and voluntary orienting of visual attention: time course of activation and resistance to interruption. Journal of Experimental Psychology: Human Perception and Performance, 15(2), 315.
Nestor, P. G., Klein, K., Pomplun, M., Niznikiewicz, M., \& McCarley, R. W. (2010). Gaze cueing of attention in schizophrenia: Individual differences in neuropsychological functioning and symptoms. Journal of Clinical and Experimental Neuropsychology, 32(3), 281-288.

Okamoto-Barth, S., \& Kawai, N. (2006). The role of attention in the facilitation effect and another inhibition of return. Cognition, 101(3), B42-B50.

Posner, M. I., \& Cohen, Y. (1984). Components of visual orienting. Attention and Performance X: Control of Language Processes, 32, 531-556.

Quadflieg, S., Mason, M. F., \& Macrae, C. N. (2004). The owl and the pussycat: Gaze cues and visuospatial orienting. Psychonomic Bulletin \& Review, 11(5), 826-831.

Ristic, J., Friesen, C. K., \& Kingstone, A. (2002). Are eyes special? it depends on how you look at it. Psychonomic Bulletin \& Review, 9(3), 507-513.

Schuller, A.-M., \& Rossion, B. (2005). Spatial attention triggered by eye gaze enhances and speeds up visual processing in upper and lower visual fields beyond early striate visual processing. Clinical Neurophysiology, 116(11), 2565-2576.

Senju, A., Tojo, Y., Dairoku, H., \& Hasegawa, T. (2004). Reflexive orienting in response to eye gaze and an arrow in children with and without autism. Journal of Child Psychology and Psychiatry, 45(3), 445-458.

Slessor, G., Laird, G., Phillips, L. H., Bull, R., \& Filippou, D. (2010). Age-related differences in gaze following: Does the age of the face matter? The Journals of Gerontology Series B: Psychological Sciences and Social Sciences, 65(5), 536-541.

Slessor, G., Phillips, L. H., \& Bull, R. (2008). Age-related declines in basic social perception: evidence from tasks assessing eyegaze processing. Psychology and Aging, 23(4), 812.

Taylor, T. L., \& Klein, R. M. (2000). Visual and motor effects in inhibition of return. Journal of Experimental Psychology: Human Perception and Performance, 26(5), 1639.

Tipples, J. (2008). Orienting to counterpredictive gaze and arrow cues. Perception \& Psychophysics, 70(1), 77-87.

Vuilleumier, P. (2002). Perceived gaze direction in faces and spatial attention: a study in patients with parietal damage and unilateral neglect. Neuropsychologia, 40(7), 1013-1026.

Weger, U. W., Abrams, R. A., Law, M. B., \& Pratt, J. (2008). Attending to objects: Endogenous cues can produce inhibition of return. Visual Cognition, 16(5), 659-674. 